\title{
Psychological Distress Associated with Patient- Reported Outcomes in Preoperative Patients with Lumbar Spinal Stenosis
}

\author{
Tomoko Kitano ${ }^{1}$, Mamoru Kawakami ${ }^{1}$, Yuyu Ishimoto ${ }^{2}$, Masatoshi Teraguchi ${ }^{1}$, \\ Daisuke Fukui ${ }^{2}$, Toshiko Matsuoka ${ }^{1}$, Yukihiro Nakagawa ${ }^{1}$

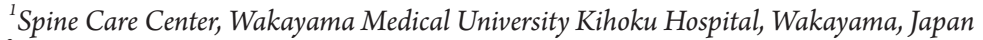 \\ ${ }^{2}$ Department of Orthopaedic Surgery, Wakayama Medical University, Wakayama, Japan
}

\section{Study Design: Cross-sectional study.}

Purpose: The purpose of this study was to investigate the effects of psychotic symptoms such as anxiety and fear in patients undergoing lumbar spinal canal stenosis.

Overview of Literature: Recently, patients with spinal disorders have not only been evaluated objectively for their disease, but also for patient-reported outcomes (PROs) including pain, physical function, and quality of life (PROs). Since depression has been previously associated with surgical outcomes, several studies have indicated that psychological problems may worsen the effects of pain and make treatment increasingly difficult.

Methods: A questionnaire survey was conducted on 346 lumbar spinal stenosis (LSS) patients who visited our hospital from 2010 to 2016. The content of the questionnaire included questions on PROs (Japanese Orthopedic Association Back Pain Evaluation Questionnaire [JOABPEQ], Roland-Morris Disability Questionnaire, Japanese version [RDQ], and Zurich Claudication Questionnaire [ZCO]) and psychological evaluation (Self-rating Questionnaire of Depression, Pain Catastrophizing Scale, Pain Anxiety Symptoms Scale-20 [PASS-20], Hospital Anxiety and Depression Scale, and Brief Scale for Psychiatric Problems in Orthopedic Patients). IBM SPSS Statistics (IBM Corp., Armonk, NY, USA) was used for statistical analysis and Spearman's rank correlation coefficient, Mann-Whitney $U$-test, and multiple regression analysis were also performed.

Results: No significant correlations were found between psychological factors and PROs $(>0.4)$. However, patients with abnormal scores for preoperative psychological items on questionnaires other than the PASS-20 also had lower scores for lumbar spine dysfunction and social life dysfunction on the JOABPEQ subscales along with higher scores for the RDQ, symptom severity and physical function on the ZCQ compared to those with normal psychological scores $(p<0.05)$.

Conclusions: Preoperative psychological factors in patients with LSS were associated with their RDQ, JOABEPQ, and ZCQ scores. These results suggest that factors such as catastrophic thoughts on pain, anxiety, depression, and fear that may affect the clinical outcomes in patients with LSS should be evaluated before surgery to facilitate psychological interventions.

Keywords: Patient reported outcome measures; Psychology; Lumbar spinal stenosis

Received Mar 5, 2020; Revised Apr 2, 2020; Accepted Apr 13, 2020

Corresponding author: Tomoko Kitano

Spine Care Center, Wakayama Medical University Kihoku Hospital, 219 Myoji, Katsuragi-cho, Ito-gun, Wakayama 649-7113, Japan

Tel: +81-736-22-0066, Fax: +81-736-22-8223, E-mail: t-kitgaw@wakayama-med.ac.jp 


\section{Introduction}

Recently, patients with spinal disorders have not only undergone objective assessments of their diseases but have also been evaluated for patient-reported outcomes (PROs) such as pain, physical function, and quality of life. Because depression has previously been associated with surgical outcomes [1], patients have undergone preoperative psychological evaluations. Previous studies have indicated that psychiatric problems are related to preoperative physical symptoms in about $13 \%$ of patients who undergo surgery [2].

Because lumbar spinal stenosis (LSS) is a chronic disease and psychological disturbances have been reported to affect surgical outcomes, there is a concern that the influence of patients' psychological status may affect their outcomes. Many studies have indicated that the psychological evaluation of patients with LSS may help to prevent low back pain from becoming chronic through early treatment of psychological problems $[3,4]$.

A previous study pointed out that psychological problems exacerbate pain and make treatment increasingly difficult. LSS not only causes pain, but also causes physical dysfunction specific to the disease itself such as lower extremity symptoms and gait disorder. In this study, we investigated pain, physical dysfunction specific to LSS, depression, and psychological problems such as anxiety and fear.

\section{Materials and Methods}

The participants in this cross-sectional study consisted of 346 patients (211 men, 135 women: consecutive patients) with LSS who were scheduled to undergo surgical intervention at Wakayama Medical University Kihoku Hospital during the period ranging from September 2010 to March 2016. All patients were evaluated preoperatively using the Roland-Morris Disability Questionnaire, Japanese version (RDQ), the Japanese Orthopedic Association Back Pain Evaluation Questionnaire (JOABPEQ), and the Japanese version of the Zurich Claudication Questionnaire (ZCQ). The RDQ asks about the degree of disability experienced during daily activities such as standing, walking, sitting, getting dressed, and working, and its score ranges from 0-24 [5]. The JOABPEQ includes five domains: painrelated disorders, lumbar dysfunction, gait disturbance, social life dysfunction, and psychological disorders. The range of score for each domain is $0-100$ points, with lower scores indicating more severe symptoms. The JOABPEQ has been shown to have a high level of reliability and validity and is often used in the evaluation of lumbar disease $[6,7]$. Scores for the ZCQ symptom-severity subscale range from 1-5 and those for the physical-function subscale range from 1-4, with higher scores indicating more severe symptoms [8].

The preoperative psychological evaluation used the Selfrating Questionnaire of Depression (SRQ-D), Hospital Anxiety and Depression Scale (HADS), Pain Catastrophizing Scale (PCS), Pain Anxiety Symptoms Scale-20 (PASS-20), and a simple questionnaire Brief Scale for Psychiatric Problems in Orthopedic Patients (BS-POP). The SRQ-D includes various questions about depressionrelated physical symptoms and is suitable for evaluating masked depression. The SRQ-D score ranges from 0-36 with higher scores indicating greater severity. A score of $\leq 10$ points is regarded as normal, a score of $10-15$ is suggestive of depression, and a score of $\geq 16$ indicates the probable presence of mild depression [9]. The SRQ-D has been found to have good reliability and validity. The HADS does not include somatic items and the presence of bodily illness does not affect the scale scores. The HADS anxiety and depression subscale scores range from 0-21, with higher scores indicating greater severity. A score of 0-7 for either subscale is regarded as normal, a score of $8-10$ is suggestive of the presence of anxiety or depression, and a score of $\geq 11$ indicates the probable presence of a mood disorder. The HADS has been found to have good reliability and validity [10]. The PCS score ranges from $0-52$, with higher scores indicating more frequent catastrophizing when experiencing pain. A total score of 0 to 31 is regarded as being in the normal range and a score of over 32 is suggestive of abnormalities. The PCS, including the Japanese version, have been shown to have sufficient internal reliability [11]. The PASS-20 score ranges from $0-100$, with higher scores indicating greater pain, anxiety, and fear of pain. A score of 0 to 14 for either subscale is regarded as being in the normal range, a score of over 15 is suggestive of abnormalities. The Japanese version of the PASS-20 has been shown to be internally reliable [12]. The BS-POP is a simpler method for evaluating psychiatric problems compared when compared to instruments such as the Minnesota Multiphasic Personality Inventory. The BS-POP consists of two parts: a questionnaire for patient evaluation which is completed by the doctor and a questionnaire related to emotions which are completed 
by the patient. The range of scores for the doctors' domain is $8-24$ points and that for the patients' domain is $10-30$ points. The cutoff values are either $>11$ points for the doctors' domain alone or $>10$ points for the doctors' domain combined with $>15$ points for the patients' domain. The BS-POP has been found to have good reliability and validity [13]. The patients' demographic data and preoperative scores for these items are shown in Table 1.

Based on the data, the relationship between PROs and the results of the psychological evaluation were first identified. Afterwards, the patients were divided into two

Table 1. Mean value and standard deviation of various tests

\begin{tabular}{|c|c|}
\hline Characteristic & Value \\
\hline No. of patients & 354 \\
\hline \multicolumn{2}{|l|}{ Gender } \\
\hline Male & 217 \\
\hline Female & 137 \\
\hline Age (yr) & $68.51 \pm 10.23$ \\
\hline RDO & $12.22 \pm 6.10$ \\
\hline \multicolumn{2}{|l|}{ JOABPEO } \\
\hline Pain related disorder & $42.28 \pm 31.80$ \\
\hline Lumbar dysfunction & $55.33 \pm 29.07$ \\
\hline Gait disturbance & $31.13 \pm 24.15$ \\
\hline Social life dysfunction & $35.18 \pm 20.67$ \\
\hline \multicolumn{2}{|l|}{ ZCO } \\
\hline Physical function & $24.19 \pm 5.27$ \\
\hline Severity of symptoms & $2.54 \pm 3.71$ \\
\hline SRQ-D & $9.79 \pm 4.74$ \\
\hline PCS total & $31.89 \pm 10.45$ \\
\hline \multicolumn{2}{|l|}{ PASS-20 } \\
\hline Escape/avoidance & $12.08 \pm 4.64$ \\
\hline Fear & $11.44 \pm 5.614$ \\
\hline \multicolumn{2}{|l|}{ HADS } \\
\hline Anxiety & $5.70 \pm 3.71$ \\
\hline Depression & $6.09 \pm 3.84$ \\
\hline \multicolumn{2}{|l|}{ BS-POP } \\
\hline Doctor version & $9.82 \pm 1.98$ \\
\hline Patient version & $16.25 \pm 3.454$ \\
\hline
\end{tabular}

Values are presented as mean \pm standard deviation.

RDO, Roland-Morris Disability Questionnaire; JOABPEO, Japanese Orthopedic Association Back Pain Evaluation Questionnaire; ZCQ, Zurich Claudication Questionnaire; SRQ-D, Self-rating Questionnaire of Depression; PCS, Pain Catastrophizing Scale; PASS-20, Pain Anxiety Symptoms Scale-20; HADS, Hospital Anxiety and Depression Scale; BS-POP, Brief Scale for Psychiatric Problems in Orthopedic Patients. groups based on whether their psychological status was regarded as normal or abnormal and evaluated whether there was a difference in the PROs. Afterwards an investigation was carried out on which psychological factors affected the preoperative PROs. This research has been approved by the Institutional Review Board of Wakayama Medical University Kihoku Hospital (IRB approva no., 2009001). Informed consent was obtained from all individual participants included in the study.

The relationships between PROs and psychological factors were analyzed using IBM SPSS Statistics ver. 23.0 (IBM Corp., Armonk, NY, USA). Spearman's rank correlation coefficient was used to evaluate the correlation between various psychological factors and PROs. Based on the cutoff values for the scores for these psychological factors, patients were divided into either a normal group or an abnormal group, and the PROs scores for each group were compared. Utilizing stepwise multiple regression analysis, we examined whether each individual PRO item (as dependent variables) was influenced at all by psychological evaluation items as independent variables. Significance was set at $|r|>0.4$ for Spearman's rank correlation coefficient, and at $p<0.05$ for the Mann-Whitney $U$-test and multiple regression analysis.

\section{Results}

The patients' age at the time of evaluation was $68.8 \pm 9.8$ years (mean \pm standard deviation). Analysis using Spearman's rank correlation coefficient revealed that there was no significant correlation between PROs and preoperative psychological evaluation items $(|r|<0.4)$ (Table 2).

Significant differences were found between the normal and abnormal groups for all PROs items except the physical-function domain of the ZCQ score (Table 3). Interestingly, even lumbar spine function which was evaluated using the lumbar spine dysfunction subscale of the JOABPEQ showed significant associations with the scores for all psychological evaluations.

Finally, Table 4 shows the results of multiple regression analysis with PROs as the independent variable and various psychological evaluations as the dependent variables. Multiple regression analysis revealed a relationship between RDQ and masked depression (SRQ-D). The analysis also revealed that JOABPEQ was associated with painrelated disorder, masked depression, pain catastrophizing, and gait disturbance. ZCQ was found to be associated 
Table 2. Correlation coefficient of various psychological evaluation and patient-reported outcomes

\begin{tabular}{|c|c|c|c|c|c|c|c|}
\hline \multirow[b]{2}{*}{ Variable } & \multirow[b]{2}{*}{ RDQ } & \multicolumn{4}{|c|}{ JOABPEQ } & \multicolumn{2}{|c|}{$\mathrm{ZCO}$} \\
\hline & & $\begin{array}{l}\text { Pain related } \\
\text { disorder }\end{array}$ & $\begin{array}{c}\text { Lumbar } \\
\text { dysfunction }\end{array}$ & $\begin{array}{c}\text { Gait } \\
\text { disturbance }\end{array}$ & $\begin{array}{c}\text { Social life } \\
\text { dysfunction }\end{array}$ & $\begin{array}{l}\text { Physical } \\
\text { function }\end{array}$ & $\begin{array}{l}\text { Severity of } \\
\text { symptoms }\end{array}$ \\
\hline SRO-D & 0.39 & -0.33 & -0.31 & -0.26 & -0.28 & 0.21 & -0.14 \\
\hline \multicolumn{8}{|l|}{ PCS } \\
\hline Total score & 0.27 & -0.15 & -0.21 & -0.25 & -0.22 & 0.25 & -0.14 \\
\hline Escape/avoidance & 0.35 & -0.24 & -0.26 & -0.24 & -0.28 & 0.19 & -0.16 \\
\hline Fear & 0.24 & -0.13 & -0.23 & -0.18 & -0.19 & 0.22 & -0.13 \\
\hline \multicolumn{8}{|l|}{ HADS } \\
\hline Anxiety & 0.26 & -0.18 & -0.31 & -0.17 & -0.21 & 0.26 & -0.18 \\
\hline Depression & 0.32 & -0.26 & -0.35 & -0.24 & -0.32 & 0.22 & -0.14 \\
\hline \multicolumn{8}{|l|}{ BS-POP } \\
\hline Doctor & 0.13 & -0.8 & -0.14 & -0.6 & -0.11 & 0.12 & -0.18 \\
\hline Patient & 0.37 & -0.38 & -0.34 & -0.23 & -0.27 & 0.2 & -0.12 \\
\hline
\end{tabular}

$p<0.05$ and $p<0.01$ are statistically significant.

RDQ, Roland-Morris Disability Questionnaire; JOABPEQ, Japanese Orthopedic Association Back Pain Evaluation Questionnaire; ZCQ, Zurich Claudication Questionnaire; SRO-D, Self-rating Questionnaire of Depression; PCS, Pain Catastrophizing Scale; HADS, Hospital Anxiety and Depression Scale; BS-POP, Brief Scale for Psychiatric Problems in Orthopedic Patients.

Table 3. Various psychological evaluation Mann-Whitney U-test in normal group/abnormal group

\begin{tabular}{|c|c|c|c|c|c|c|c|c|c|}
\hline \multirow[b]{2}{*}{ Variable } & \multirow[b]{2}{*}{ Group } & \multirow[b]{2}{*}{ No. } & \multirow[b]{2}{*}{ RDQ } & \multicolumn{4}{|c|}{ JOABPEQ } & \multicolumn{2}{|c|}{$\mathrm{ZCO}$} \\
\hline & & & & $\begin{array}{l}\text { Pain related } \\
\text { disorder }\end{array}$ & $\begin{array}{l}\text { Lumbar } \\
\text { dysfunction }\end{array}$ & $\begin{array}{c}\text { Gait } \\
\text { disturbance }\end{array}$ & $\begin{array}{c}\text { Social life } \\
\text { dysfunction }\end{array}$ & $\begin{array}{l}\text { Physical } \\
\text { function }\end{array}$ & $\begin{array}{l}\text { Severity of } \\
\text { symptoms }\end{array}$ \\
\hline SRQ-D $\leq 10$ & Normal & 206 & $10.8 \pm 6.6$ & $51.6 \pm 31.7$ & $62.1 \pm 28.2$ & $35 \pm 25.2$ & $39.4 \pm 20.8$ & 3.3 & 0.4 \\
\hline SRQ-D $>11$ & Abnormal & 126 & $14.8 \pm 4.4^{* *}$ & $28.8 \pm 28.4^{* *}$ & $43.4 \pm 27.6^{* *}$ & $22.2 \pm 19.1^{* *}$ & $27.2 \pm 19.2^{* *}$ & $3.6^{* *}$ & $0.2^{* *}$ \\
\hline PCS total $\leq 32$ & Normal & 161 & $11.5 \pm 7.3$ & $45.2 \pm 33.3$ & $58.6 \pm 29.4$ & $35.1 \pm 25.2$ & $38 \pm 22.3$ & 3.4 & 1.4 \\
\hline PCS total $>33$ & Abnormal & 169 & $3.1 \pm 4.8^{*}$ & $39.2 \pm 30.3$ & $52.1 \pm 28.9^{*}$ & $26.1 \pm 21.9^{* *}$ & $31.7 \pm 18.8^{*}$ & $3.5^{*}$ & $0.3^{*}$ \\
\hline PASS-20 (escape/avoidance) $\leq 15$ & Normal & 227 & $11.5 \pm 6.4$ & $46.5 \pm 31.7$ & $59.4 \pm 28.2$ & $32.7 \pm 23.6$ & $38 \pm 20.5$ & 3.4 & 0.3 \\
\hline PASS-20 (escape/avoidance) > 16 & Abnormal & 87 & $14.4 \pm 5^{* *}$ & $30.9 \pm 29.9^{* *}$ & $43.7 \pm 29.1^{* *}$ & $24.3 \pm 24.1^{*}$ & $26.3 \pm 19.8^{* *}$ & $3.6^{*}$ & 0.3 \\
\hline PASS-20 (fear) $\leq 15$ & Normal & 219 & $11.3 \pm 5.2$ & $44.9 \pm 32.5$ & $58.6 \pm 29$ & $32 \pm 23.1$ & $37 \pm 21$ & 3.4 & 0.3 \\
\hline PASS-20 (fear) $>16$ & Abnormal & 95 & $4.6 \pm 7.6^{*}$ & $36.4 \pm 29.9^{*}$ & $47.2 \pm 28.4^{*}$ & $26.7 \pm 25.6^{*}$ & $30 \pm 20^{*}$ & $3.6^{*}$ & 0.3 \\
\hline HADS anxiety $\leq 8$ & Normal & 207 & $11.6 \pm 6.6$ & $44.2 \pm 32.4$ & $59.6 \pm 28.5$ & $31.8 \pm 23.9$ & $36.6 \pm 20.8$ & 3.3 & 0.4 \\
\hline HADS anxiety $>9$ & Abnormal & 102 & $13.7 \pm 5.1$ & $38.8 \pm 31.3$ & $45.3 \pm 29 * *$ & $27.5 \pm 24.4$ & $30.4 \pm 20.6^{*}$ & $3.7^{* *}$ & $0.2^{*}$ \\
\hline HADS depression $\leq 8$ & Normal & 199 & $11.4 \pm 6.5$ & $48.4 \pm 32.1$ & $62.1 \pm 26.6$ & $32.4 \pm 23.9$ & $38.6 \pm 20.6$ & 3.3 & 0.4 \\
\hline HADS depression $>9$ & Abnormal & 110 & $4.1 \pm 5.2^{*}$ & $31.5 \pm 29.2^{* *}$ & $41.6 \pm 29.8^{*}$ & $26.6 \pm 24.2^{*}$ & $27.1 \pm 19.5^{* *}$ & $3.6^{* *}$ & $0.2^{*}$ \\
\hline BS-POP for spinal surgery $\leq 10, \leq 8$, or $\leq 15$ & Normal & 182 & $11.7 \pm 6.7$ & $46.6 \pm 32.6$ & $59.4 \pm 28.6$ & $31.6 \pm 24.8$ & $37.8 \pm 21.2$ & 3.3 & 0.4 \\
\hline BS-POP for spinal surgery $>10,>8$, or $>15$ & Abnormal & 140 & $13.1 \pm 5.3$ & $37.8 \pm 31^{*}$ & $49.8 \pm 29.8^{*}$ & $28.4 \pm 22.8$ & $31 \pm 19.8^{*}$ & $3.5^{*}$ & $0.3^{*}$ \\
\hline
\end{tabular}

Values are presented as mean \pm standard deviation, unless otherwise stated.

RDQ, Roland-Morris Disability Questionnaire; JOABPEQ, Japanese Orthopedic Association Back Pain Evaluation Questionnaire; ZCQ, Zurich Claudication Questionnaire; SRQ-D, Self-rating Questionnaire of Depression; PCS, Pain Catastrophizing Scale; PASS-20, Pain Anxiety Symptoms Scale-20; HADS, Hospital Anxiety and Depression Scale; BS-POP, Brief Scale for Psychiatric Problems in Orthopedic Patients.

" $p<0.05 . " p<0.01$. 
Table 4. Multiple regression analysis with patient-reported outcomes as independent variable and various psychological evaluations as dependent variables

Variable

With $\mathrm{RDQ}$ as a dependent variable

\begin{tabular}{|cc|}
\hline SRQ-D & $0.198^{*}$ \\
\hline BS-POP (patient version) & $0.215^{*}$ \\
\hline With JOABPEQ pain-related disorder as a dependent variable & \\
\hline SRQ-D & $-0.168^{*}$ \\
\hline BS-POP (patient version) & $-0.323^{* *}$ \\
\hline With JOABPEQ lumbar spine dysfunction as a dependent variable & \\
\hline HADS depression & $-0.383^{* *}$
\end{tabular}

With JOABPEO gait disturbance as a dependent variable

\begin{tabular}{cc} 
PCS total & $-0.234^{*}$ \\
\hline HADS depression & $-0.223^{* *}$ \\
With JOABPEO social life dysfunction as a dependent variable & \\
\hline HADS depression & $-0.326^{* *}$ \\
With ZCQ physical function as a dependent variable & \\
\hline HADS anxiety & $-0.24^{* *}$ \\
With ZCQ severity of symptoms as a dependent variable & \\
\hline PCS magnification & $-0.167^{*}$
\end{tabular}

RDQ, Roland-Morris Disability Questionnaire; SRQ-D, Self-rating Questionnaire of Depression; BS-POP, Brief Scale for Psychiatric Problems in Orthopedic Patients; JOABPEQ, Japanese Orthopedic Association Back Pain Evaluation Questionnaire; HADS, Hospital Anxiety and Depression Scale; PCS, Pain Catastrophizing Scale; ZCQ, Zurich Claudication Questionnaire. $p<0.05 . * 0<0.01$

with ZCQ physical function and HADS anxiety, ZCQ symptom severity, and PCS magnification.

\section{Discussion}

The preoperative PROs of patients with LSS were evaluated using various psychological evaluation questionnaires as part of a future prospective study. Psychological factors including pain catastrophizing, anxiety, and depression in patients with LSS before surgery were associated with RDQ, JOABPEQ, and ZCQ scores, which have been used for the evaluation of low back pain and LSS.

We found that this preoperative psychological distress was also related with lumbar spine dysfunction as evaluated by the JOABPEQ, although the JOABPEQ questions are more or less related to evaluation of lumbar function.

Furthermore, from the results of this multiple regression analysis, it may be possible that catastrophic thinking, such as being unable to do anything, is affecting gait function. In addition, since ZCQ function has been significantly associated with anxiety, it is thought that strong anxiety may lead to a decline in physical function. Additionally, ZCQ symptom severity was associated with magnified pain and a tendency to overstate the symptoms seemed to affect its severity.

It is speculated that patients' fear of physical activity and anxiety about their pain and physical health continuing to deteriorate may end up leading to dysfunction of the lumbar spine. It is also possible that anxiety and fear related to preoperative feelings about their symptoms could be exacerbated and thoughts on the surgery being ineffective might affect the preoperative PROs. Psychological factors such as fear and anxiety, including the fear avoidance model, may lead to decreased motivation for activity and are thought to be the cause of depression that is associated with chronic symptoms of LSS. It is speculated that psychological intervention before surgery may be necessary to prevent the psychological deterioration of a patient's physical symptoms. It is also cited that psychological factors also influence surgical results and patient satisfaction with surgery [14]. Therefore, it is possible that relieving not only depression, but also fear, anxiety, and pain catastrophizing thoughts before surgery may result in improved clinical outcomes. Following these results, it may be worth conducting future research on the subject to clarify how preoperative psychological interventions affect the patient's postoperative course and patient satisfaction.

Some limitations of this included not evaluating the duration of symptoms prior to surgery, comorbidities of the patients, the patients' background, and living conditions all of which may have affected their psychological well-being. Although it was found that preoperative psychological factors were significantly associated with lumbar spine function, it could not be determined whether or not this was a causal association due to the cross-sectional study design. In the future, it is advised that prospective studies should consider unifying patient selection and evaluation time.

\section{Conclusions}

The study examined the relationship between PROs and psychological factors in 346 patients with LSS before surgery. Significant differences were found between the psychologically normal and abnormal groups for scores 
on the RDQ, the lumbar spine dysfunction and social life dysfunction subscales of the JOABPEQ, and symptom severity and physical function in the ZCQ. Additionally, scores on the gait subscale of the JOABPEQ and physical function as assessed by ZCQ were related to the PCS score and the anxiety score in HADS. Since preoperative psychological factors in patients with LSS are significantly related to PROs, it is necessary to assess psychological factors as part of patients' preoperative examinations.

\section{Conflict of Interest}

No potential conflict of interest relevant to this article was reported.

\section{References}

1. Sinikallio S, Aalto T, Airaksinen O, et al. Depression is associated with poorer outcome of lumbar spinal stenosis surgery. Eur Spine J 2007;16:905-12.

2. Sato K, Kikuchi J, Ootani K. Evaluation of psychiatric problems in relation to surgical treatment for degenerative lumbar spinal diseases. Clin Orthop Surg 2004;39:1145-50.

3. Carragee EJ. Psychological and functional profiles in select subjects with low back pain. Spine J 2001;1:198-204.

4. Simmonds MJ, Kumar S, Lechelt E. Psychological factors in disabling low back pain: causes or consequences? Disabil Rehabil 1996;18:161-8.

5. Suzukamo Y, Fukuhara S, Kikuchi S, et al. Validation of the Japanese version of the Roland-Morris Disability Questionnaire. J Orthop Sci 2003;8:543-8.
6. The Japanese Society for Spine Surgery and Related Research. Manual of JOABPEQ JPACMEQ. Tokyo: Nankodo; 2012.

7. Miyamoto M, Fukui M, Konno S, et al. Development of new disease-specific QOL measure for patients with low back pain: JOA Back Pain Evaluation Questionnaire (JOABPEQ). J Jpn Soc Lumbar Spine Disord 2009;15:23-31.

8. Hara N, Matsudaira K, Masuda K, et al. Psychometric assessment of the Japanese version of the Zurich Claudication Questionnaire (ZCQ): reliability and validity. PLoS One 2016;11:e0160183.

9. Rockliff BW. A brief self-rating questionnaire for depression (SRQ-D). Psychosomatics 1969;10:236-43.

10. Hatta H, Higashi A, Yashiro H, et al. A validation of the Hospital Anxiety and depression Scale. Jpn J Psychosom Med 1998;38:309-15.

11. Matsuoka H, Sakano Y. Assessment of cognitive aspect of pain: development, reliability, and validation of Japanese version of pain catastrophizing scale. Jpn J Psychosom Med 2007;47:95-102.

12. Matsuoka H, Sakano Y. Development and validation of Japanese version of Pain Anxiety Symptoms Scale-20. Jpn J Behav Med 2009;14:1-7.

13. Watanabe K, Kikuchi S, Konno S, Niwa S, Masiko H. Brief Scale for Psychiatric Problems in Orthopaedic Patients (BS-POP) validation study. Clin Orthop Surg 2005;40:745-51.

14. Yoshida K, Sekiguchi M, Otani K, et al. A validation study of the Brief Scale for Psychiatric problems in Orthopaedic Patients (BS-POP) for patients with chronic low back pain (verification of reliability, validity, and reproducibility). J Orthop Sci 2011;16:713. 